\title{
Régulations en rivière
}

\section{P. Hurand}

Cie d'Aménagement des Côteaux de Gascogne

\section{P. Kosuth}

CEMAGREF
De nombreux systèmes hydrauliques pour l'irrigation (et aussi l'eau potable) utilisent les cours d'eaux naturels pour acheminer l'eau depuis un barrage amont jusqu'aux zones de consommation (périmètres irrigués, réseaux de distribution d'eau potable alimentés par des pompages au fil de l'eau). Ces systèmes sont très répandus en Europe, en Afrique du Nord et en Amérique du Sud. En France, c'est dans le Sud-Ouest qu'on les retrouve essentiellement. La CACG gère ainsi, en tant que concessionnaire de l'Etat ou fermier des collectivités locales, la réalimentation d'une vingtaine de rivières: ce sont les affluents gascons de la Garonne ou bien des rivières du bassin de l'Adour. Le linéaire de cours d'eaux ainsi réalimentés est voisin de $2000 \mathrm{~km}$, la surface irriguée atteint 50000 ha et près de 200000 usagers dépendent de ces rivières pour leur alimentation en eau potable. La réalimentation est effectuée à partir du canal de la Neste et d'une vingtaine de barrages dont la capacité varie de $25 \mathrm{hm}^{3}$ à moins du million de $\mathrm{m}^{3}$ pour les plus petits.

Après un rappel succinct des caractéristiques hydrauliques des rivières ainsi réalimentées et des objectifs de gestion, on présentera les différents outils utilisés en matière de réalimentation, que ce soit le système permettant de connaître, en temps réel, les débits en différents points d'une rivière et d'effectuer des commandes à distance, ou les logiciels qui simulent le fonctionnement hydraulique ou calculent automatiquement la lâchure à effectuer depuis le barrage amont. Enfin on essaiera, grâce à l'expérience acquise depuis maintenant une dizaine d'années, d'évaluer les apports et les limites de cette forme de gestion " automatisée ".

\section{Rivières du Sud-Ouest et gestion des eaux}

\subsection{Contexte hydraulique et hydrologique}

Les rivières réalimentées par la Compagnie d'aménagement des Côteaux de Gascogne (CACG) présentent un certain nombre de caractéristiques communes :

- leur longueur n'excède pas $150 \mathrm{~km}$, ce qui représente des temps de transfert, entre le barrage et l'aval, ne dépassant pas 4 jours ;

- les apports naturels sont, sinon nuls du moins négligeables par rapport aux prélèvements effectués pendant la campagne d'irrigation, donnant des types d'écoulement voisins de ceux qu'on trouve dans les canaux (décroissance des débits d'amont vers l'aval);

- le seul point de commande se situe à l'amont, à la vanne du barrage : la gestion correcte de ces systèmes "barragerivière-stations de pompage " repose sur la détermination du débit à lâcher au barrage ; en particulier on ne dispose pas de vannes intermédiaires partageant la rivière en biefs, et permettant de jouer, comme c'est le cas sur certains canaux, sur le volume du bief amont pour alimenter les parties aval ;

- la période de réalimentation commence en début juillet (exceptionnellement mi-juin). Elle peut se poursuivre bien au-delà de la période d'irrigation jusqu'en décembre, voire janvier ou février.

De plus, la fourniture d'eau s'effectue à la demande, c'est-à-dire que chaque utilisateur prend l'eau quand il en a besoin, sans contrainte de tour d'eau programmé.

\section{River regulation}

In SW-France, irrigation and water supply generally use direct pumping in rivers. The upstream flow is regulated through a dam. The management of these hydraulic systems is geared to satisfying water demands while complying with the downstream flow orders and saving water as much as possible $\star$ Such a management can be optimized thanks to the existing simulation software as well as methods of automatics ; the former leads to a correct modelisation of the hydraulic system operation. It becomes therefore possible to get a better knowledge of its overall dynamics and to understand interactions between inflow, withdrawals and various perturbations. Once such an understanding achieved, technics of automatics help to determine the optimum orders to be carried out at the dam, as well as to detect the perturbations and react to them. Testing and improving regulation can be done through the simulation tools. 


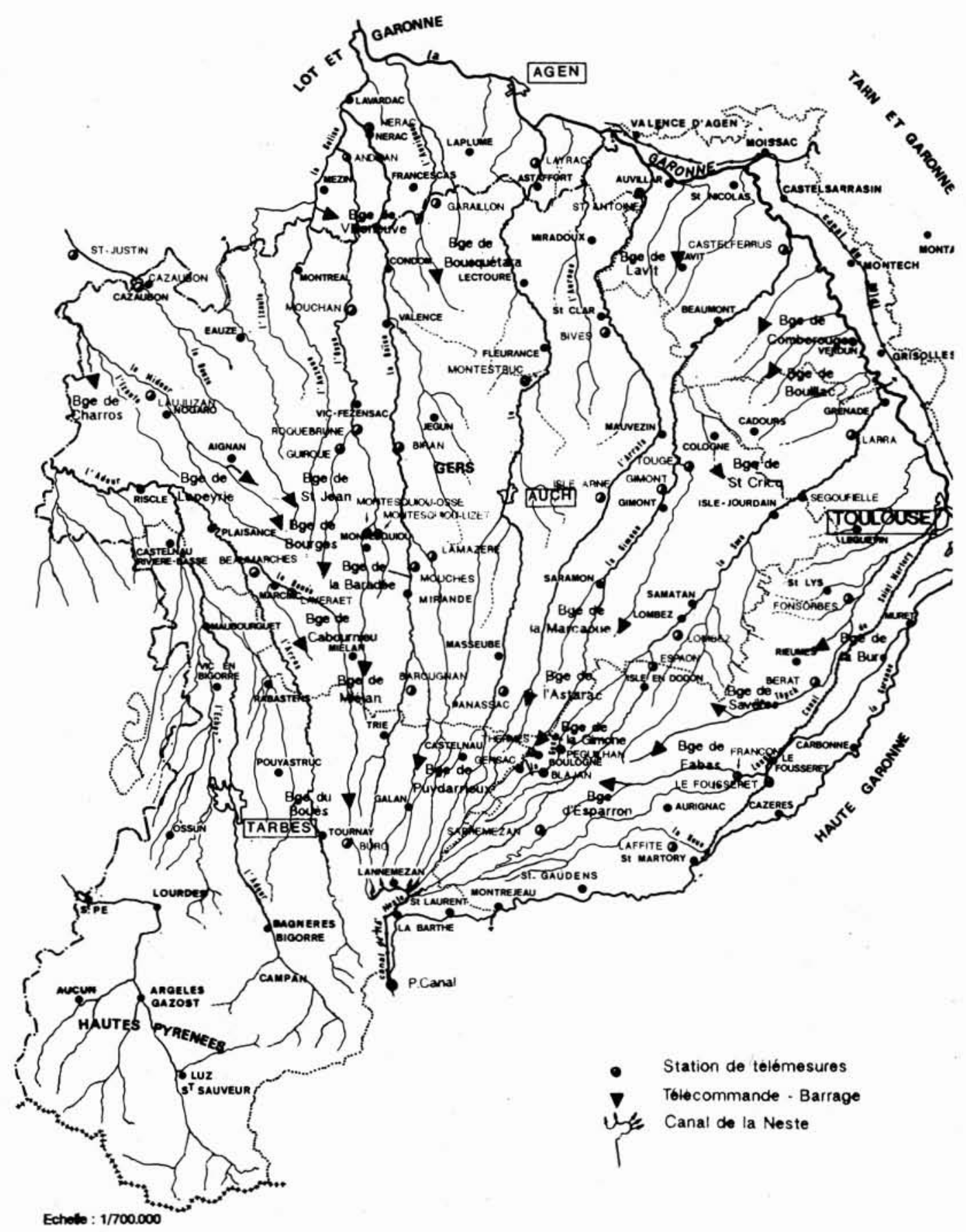

1. Réseau de télégestion CACG.

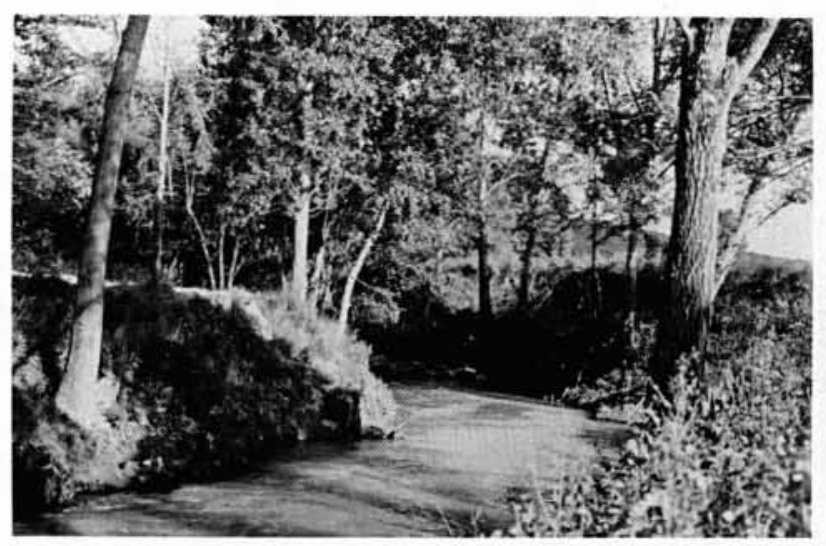

2. Rivière Arrats.

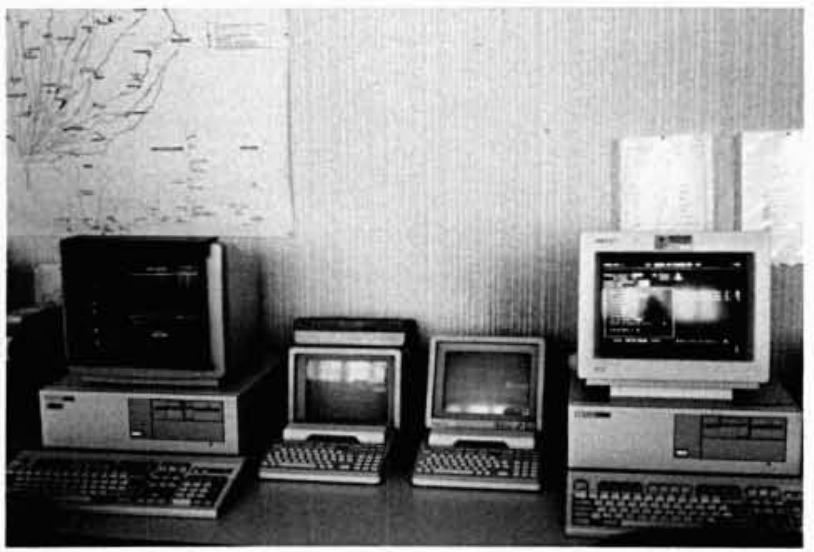

3. Centre de Gestion de Tarbes. 


\section{RÉGULATIONS EN RIVIÈRE}

Ajoutons enfin qu'on se situe dans un contexte où le bilan " besoins-ressources " est à peine équilibré, c'est-àdire où l'eau gaspillée risque de faire défaut en fin de campagne.

\subsection{Les objectifs de gestion}

Au départ, l'objectif de gestion est double; il consiste : - à fournir à chaque préleveur (irrigant, syndicat d'alimentation en eau potable), à tout moment, l'eau dont il a besoin ;

- à s'assurer qu'il reste en tout point du cours d'eau un débit suffisant pour préserver sa salubrité : compte tenu du contexte hydrologique spécifique de ces cours d'eau, cet objectif se ramène le plus souvent à conserver un débit donné à l'aval du cours d'eau. Ce débit peut être modulé à un pas de temps en général hebdomadaire, en fonction de l'état du stock d'eau dans les réservoirs, des prévisions sur les prélèvements à venir... Le calcul de ces modulations fait appel aux techniques de la programmation dynamique ; il constitue la base de ce qu'on appelle la gestion stratégique d'un système hydraulique réalimenté. En matière de régulation de lâchures proprement dite (qu'on a d'ailleurs coutume, par opposition, d'appeler " gestion tactique »), c'est une donnée au même titre que les prélèvements.

En corollaire à ce double objectif, compte tenu du contexte général de pénurie, on cherche à respecter de très près ces consignes de débit car, si un débit inférieur à la consigne peut être considéré comme une défaillance, tout excédent est assimilable à une perte d'eau. Pour cela, il est nécessaire de prévoir les prélèvements et de lâcher à l'avance les débits correspondants pour qu'ils arrivent au bon endroit, au bon moment. Il faut également réagir à des perturbations affectant le système (pluies, mise en route ou arrêt imprévus des stations de pompage). La performance d'un système de régulation va ainsi exiger :

- d'avoir accès en temps réel à l'information (débits injectés à partir d'un barrage, débits au droit du point de consigne, mais aussi mesures intermédiaires...);

- de connaître parfaitement le système hydraulique (temps de retard, prélèvements, ...);

- de choisir une méthode permettant de réagir aux perturbations détectées.

\section{Les outils d'une bonne régulation}

On présentera ici la démarche développée par la CACG (avec l'aide du CEMAGREF pour la partie "modélisation ») et qui peut se décomposer en trois parties:

- mise en place d'un système d'acquisition de l'information et de transmission des commandes en temps réel ; - utilisation d'un modèle simple de simulation (BAHIA), permettant d'effectuer des calculs dans un temps réduit ; - application des techniques de l'automatique pour la gestion des systèmes hydrauliques.
Le système d'acquisition de l'information et de transmission de commandes en temps réel est l'outil de base préalable et indispensable à toute modernisation et amélioration de la gestion. A lui seul, il peut en améliorer considérablement les performances.

Le modèle de simulation permet de mieux comprendre la dynamique du système hydraulique (effet des prélèvements sur le transfert de débits, influence des lâchures effectuées au barrage, variation des temps de transfert selon l'état de la rivière, ...), de tester des méthodes de gestion (gestion manuelle classique ou régulation automatique) et de les améliorer avant de les mettre en œuvre sur le système réel.

L'automatique permet d'optimiser les commandes au barrage en fonction des objectifs définis plus haut et des informations disponibles en temps réel sur le système. Elle présente l'intérêt d'améliorer les performances hydrauliques, d'économiser l'eau et de libérer les opérateurs du travail de routine pour se consacrer aux tâches de supervision et aux contacts avec les utilisateurs.

\subsection{Le système d'acquisition de l'information et de trans- mission des commandes en temps réel}

Il se compose, à la CACG :

- d'un serveur "réseau " et de trois micro-ordinateurs compatibles AT reliés au serveur ; l'un est quasiment dédié à l'acquisition de données et à l'envoi automatique de commandes, les deux autres permettant de visualiser, d'analyser et d'interpréter les données;

— d'un parc de près de 200 stations de télémesure (débits rivière, niveau des réservoirs, oxygène dissous, température...) équipées soit de sondes (pression, température, ...) soit de codeurs optoélectroniques installés sur des limnigraphes ;

- d'une trentaine de prises sur canal ou vannes de barrages motorisées, automatisées et télécommandables à distance.

Les liaisons « PC-stations » se font à l'aide d'une carte modem, via le réseau auto-commuté de "FranceTélécom ». L'interrogation et l'envoi des commandes peuvent se faire à tout moment, à la demande, à l'aide d'un des micro-ordinateurs ou d'un simple minitel. Généralement, ils se font de façon automatique par microordinateur suivant des cycles d'interrogation ou de commandes programmables, donc modifiables, en fonction de la densité de l'information jugée nécessaire. C'est ainsi que les stations donnant le niveau des réservoirs seront interrogées une fois par jour, alors que les stations donnant le débit sur les rivières réalimentées le seront à des pas de temps variant de une à quelques heures.

\subsection{Le modèle simple de simulation}

- Le phénomène hydraulique à modéliser est, a priori, complexe :

- on s'intéresse à des modulations de lâchures, donc à des phénomènes d'écoulement en régime transitoire; 
Rivière ARRATS (France Sud-Ouest)

Comparaison HAYAMI / 2ème ordre retardé sur un créneau de débit

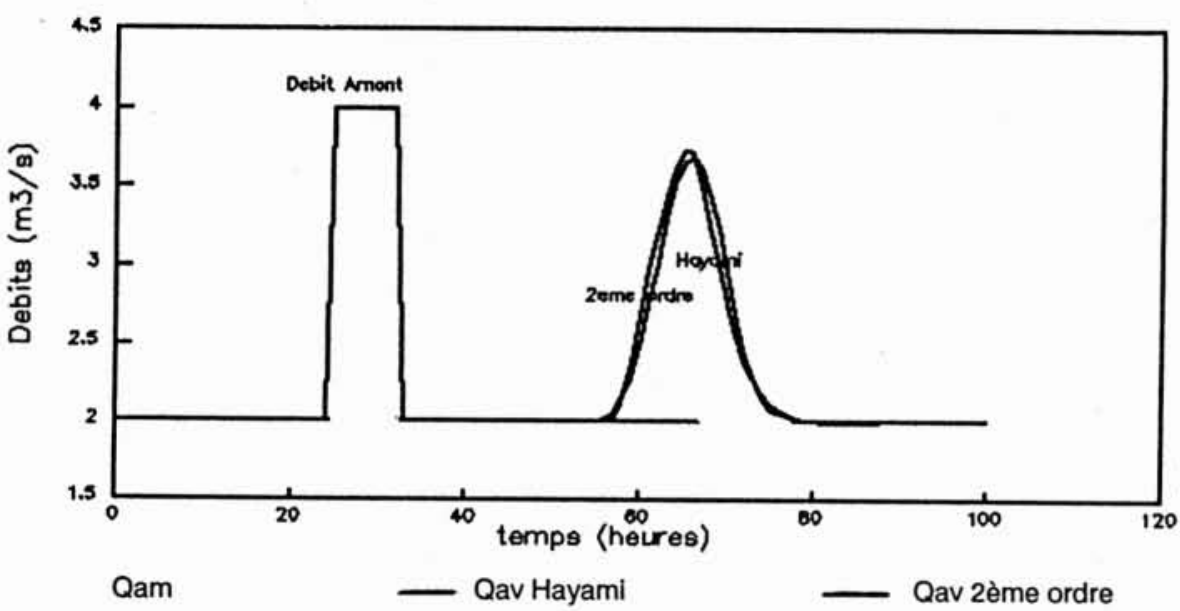

Longueur totale $109 \mathrm{~km}$, teta $=0,34 \mathrm{~h} / \mathrm{km}, \mathrm{Z}=0,861 / \mathrm{km}$

$r=33 h, a=1,407, b=0,512, c=0,058, d=0,047$

4. Représentation de la dynamique par un système simplifié du 2 ordre.

Rivière ARRATS (France Sud-Ouest) / simulation Régul. par placement de pôles. Pluies et arrêt des prélèvements

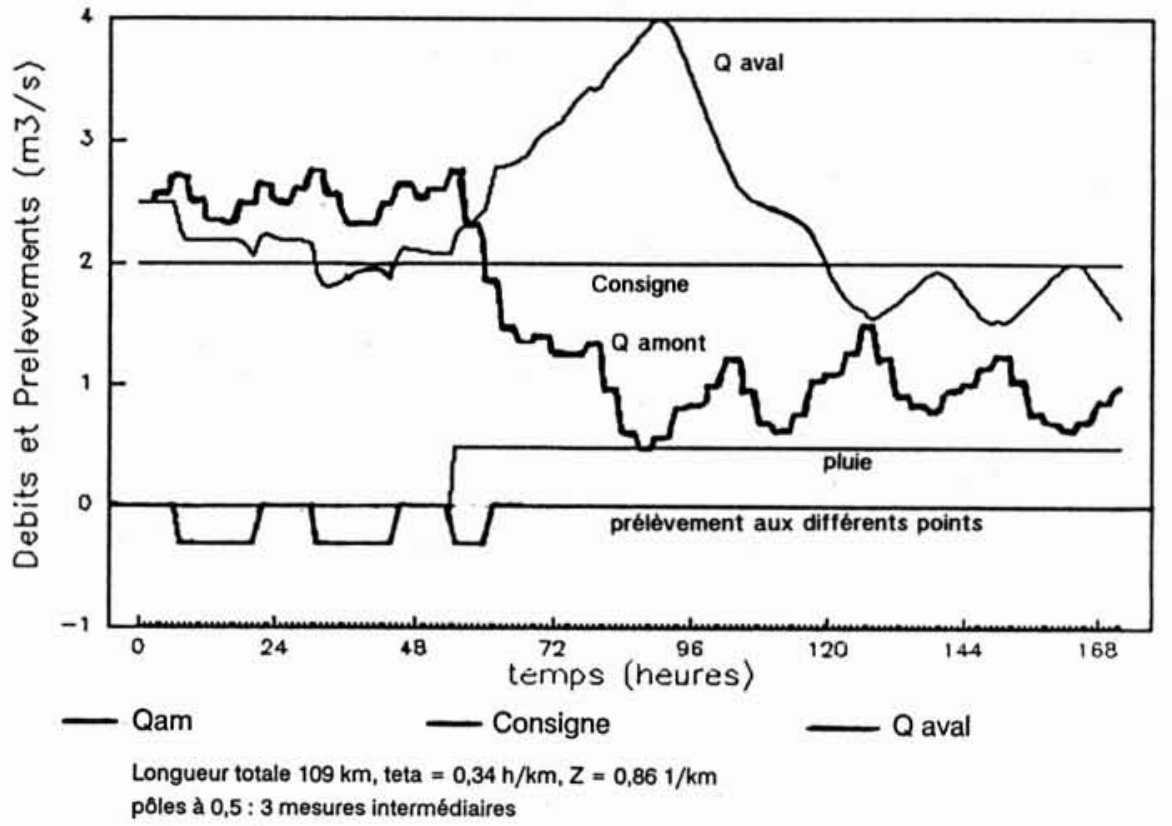

5. Régulation par placement de pôles dans le cas de pluies imprévues provoquant l'arrêt des irrigations.

- les débits des lâchures sont eux-mêmes fortement perturbés par des apports naturels difficiles à estimer et surtout par des prélèvements qu'on ne sait évaluer que de façon approximative et globale (si on connait toutes les pompes, on ne sait jamais avec précision lesquelles sont en fonctionnement) ; 
- la longueur des cours d'eau réalimentés peut être importante (de l'ordre de la centaine de kilomètres).

La résolution classique de ce type de problème est à la fois exigeante en données (notamment sur la géométrie du lit de la rivière) et en temps de calcul, deux contraintes difficilement compatibles avec les objectifs recherchés de rapidité de calcul et de simplicité de mise en œuvre.

- Le logiciel BAHIA évite de tels inconvénients. La description du système hydraulique est rapide: on se contente de positionner les nœuds hydrauliques (points de confluence, de prélèvements et de mesures); la simulation du fonctionnement repose sur la modélisation des transferts d'hydrogrammes par résolution de l'équation d'Hayami qui décrit la dynamique de la rivière à l'aide des deux paramètres: temps de retard et paramètre de diffusion. Des modules de calage intégrés dans le logiciel BAHIA permettent de déterminer ces paramètres à partir des mesures de terrain.

Disposant de cette représentation du système, il est possible de simuler n'importe quel scénario hydraulique : changement des lâchures au barrage, mise en route d'une station de pompage, arrivée d'une pluie et arrêt des prélèvements d'irrigation... Pour cela on introduit, de manière conversationnelle, les éléments correspondant au scénario.

L'application la plus immédiate est d'ordre pédagogique : c'est celle qui permet au gestionnaire de tester ses réactions dans quelques cas simples et bien caractéristiques. L'utilisation, comme outil d'interpretation et de compréhension de phénoménes observés sur le terrain est, quant à elle, plus problématique; en tout cas elle est rarement immédiate, l'origine des perturbations étant souvent multiple : elle requiert de la part de l'utilisateur beaucoup d'expérience. Enfin, ce logiciel s'avère très pratique pour tester et améliorer l'efficacité des algorithmes de régulation automatique.

\subsection{L'automatique appliqué à la commande des systèmes hydrauliques}

L'automatique, ou analyse des systèmes, est une méthode très générale permettant d'étudier la dynamique et la commande des systèmes.

\section{a) La modélisation linéaire}

Le système est représenté par une "boîte noire " soumise à des entrées (actions extérieures: débit amont, prélèvements, ...) et fournissant des sorties (résultats de ces actions extérieures: débit aval, débits ou niveaux en différents points du système, ...). L' "intérieur » de la boîte noire est représenté par un modèle simple, linéaire, reliant entrées et sorties. Sur ce type de systeme, les informations sont mesurées à un certain pas de temps $T$ (demi-heure, heure, ...) et l'on adopte un modèle linéaire qui traduit la relation entre le débit à l'aval d'une rivière aux instants $t, t-T, T-2 T$ et :

- le débit injecté du barrage aux instants $t-r T$, $t-(r+1) T, t-(r+2) T$;
- les prélèvements effectués aux instants $t-r h T$, $T-(r h+1) T, t-(r h+2) T$ où $T$ est la période de mesure, $r T$ et $r h T$ les retards du système $(0<h<1)$.

La figure 4 illustre la fiabilité de ce type de relation par rapport au modèle d'Hayami.

Deux types de méthodes peuvent être employés pour effectuer le calage du modèle linéaire (communément appelé " $2^{c}$ ordre ") :

- soit on procède à un ajustement direct du modèle linéaire sur les données de terrain,

- soit on effectue un calcul analytique des coefficients du modèle linéaire en fonction des paramètres d'Hayami, luimême ajusté sur des données de terrain.

\section{b) Le calcul de la commande}

- Plusieurs méthodes de régulation automatique sont envisageables pour asservir la commande (la lâchure du barrage) aux prévisions et aux valeurs de débit mesurées en différents points du système. Quelle que soit la méthode de régulation, la commande est décomposée en une somme de deux termes:

- un premier terme dit "boucle ouverte" uniquement fonction de l'objectif de débit à l'aval et des prévisions de prélèvements ;

- un deuxième terme dit "boucle fermée " qui opère une correction de la boucle ouverte en fonction des écarts constatés entre le calcul théorique donné par la boucle ouverte et les mesures effectuées en temps réel sur le système.

A partir de ce même principe, il existe plusieurs types de régulation qui diffèrent selon la façon:

- "d'inverser » la fonction de transfert (calcul en boucle ouverte) ;

— de prendre en compte les écarts constatés.

La CACG applique, pour sa part, une méthode de régulation dite "par placement de pôles " garantissant la stabilité et la précision du système régulé.

La stabilité est la qualité fondamentale des systèmes de régulation automatique. Dans le cas présent, c'est elle qui nous assure que d'une commande à l'autre, les vannes ne vont pas alternativement s'ouvrir en grand ou se fermer. Imaginons en effet une perturbation cyclique de période courte (prélèvement imprévu ou éclusée de moulin) située juste à l'amont d'un point de mesure. Si le temps de transfert est supérieur à la période du cycle de la perturbation, la correction de lâchures effectuée sera en général inutile; elle risque même d'amplifier le phénomène. Pour éviter ce risque, la prise en compte des perturbations n'est que progressive : on prend de plus en plus la perturbation en compte au fur et à mesure qu'elle se confirme au cours des cycles de commande. La stabilité est donc assurée par un "lissage " adéquat des perturbations constatées.

Cette opération de "lissage " ne doit pas changer en moyenne les volumes lâchés depuis le barrage. Il en est de même dans le calcul en boucle ouverte, où les volumes lâchés doivent correspondre aux volumes des besoins: le système de commande est dit, dans ces conditions, précis. 


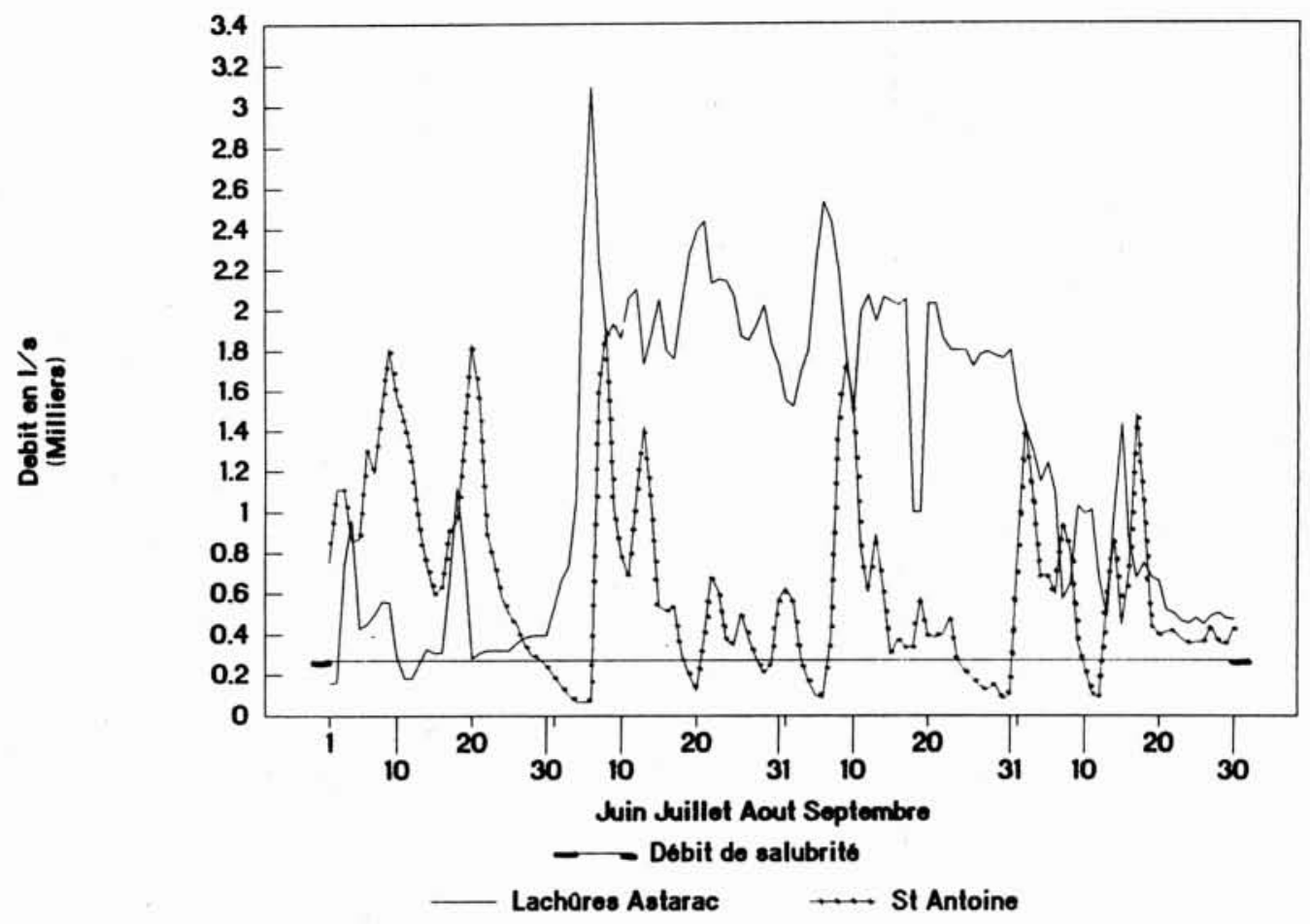

6. Gestion de l'Arrats. Bilan de la campagne 91.

\section{Gestion en temps réel. Réflexions sur 10 ans de pratique}

On regroupe sous le terme de gestion en temps réel les deux notions développées au paragraphe précédent, à savoir :

- acquisition d'informations et envoi de commandes en temps réel ;

— régulation automatique des lâchures de barrages.

Elles sont, en effet, à la CACG, indissociables car historiquement mises en œuvre de façon simultanée: on peut même dire que la mise en place des systèmes d'acquisition d'information et d'envoi de commande en temps réel a résulté de la volonté de mettre en place un dispositif de régulation automatique des lâchures de barrages. La comparaison se fait avec les modes de gestion traditionnelle où la connaissance des débits se fait grâce aux relevés périodiques des limnigraphes et où la modification des lâchures d'un barrage nécessite d'aller sur place manœuvrer la vanne.

\subsection{Des gains indiscutables}

Ils peuvent se résumer par la formule :

"Amélioration de l'efficience sans remise en cause de la sécurité des approvisionnements »
Mais d'abord qu'appelle-t-on " efficience » ? En fait, ce terme peut recouvrir deux formulations mathématiques :

— traditionnellement l'efficience est donnée par la formule :

$$
e=1-\Sigma \frac{(\text { Qmesuré }- \text { Consigne })}{\text { Volume lâché }}
$$

l'efficience vaut 1 si la consigne a été respectée en moyenne. Cette notion est intéressante lorsqu'on est amené à faire des bilans en volume " besoins-ressources";

- par contre, si on veut juger de la performance du système de régulation, on applique la formule :

$$
e=1-\Sigma \frac{(\text { Qmesuré }- \text { Consigne })}{\text { Volume lâché }}
$$

on peut alors parler d'indice de performance.

Actuellement, on arrive sur des rivières comme l'Arrats ou la Baïse, où le volume des restitutions se situe entre 10 et $15 \mathrm{hm}^{3}$, à des pourcentages de pertes de l'ordre de $20 \%$, soit une efficience de $80 \%$, pour des indices de performances de l'ordre de $75 \%$.

Avec les systèmes traditionnels, ces mêmes indices étaient de l'ordre de 50 à $60 \%$ : la gestion en temps réel permet ainsi une économie d'eau de $20 \%$. Notons bien que cette amélioration de l'efficience s'est faite sans augmentation des risques de manque d'eau à l'aval, comme le montre d'ailleurs la figure 6. 


\subsection{Quelles perspectives d'amélioration?}

Elles passent par la résolution des problèmes qu'on rencontre encore aujourd'hui et qu'on s'efforce de résoudre progressivement.

Ces problèmes concernent à la fois la théorie et la pratique.

Sur le plan théorique, si le modèle d'Hayami se prête bien à la linéarisation, donc à des calculs rapides, ses approximations restent assez grossières. Une des plus gênantes est, sans conteste, l'indépendance des temps de transfert du débit. Les calages de ces temps de transfert, indispensables au fonctionnement du modèle, ne sont donc bons que pour une configuration de débit donnée, ce qui impose d'adoucir encore davantage les réactions de la boucle fermée pour couvrir les autres cas de figure. Des travaux sont actuellement en cours pour prendre en compte cette variation des temps de transfert sans avoir recours à des modẻles compliqués nécessitant des temps de calcul incompatibles avec la gestion en temps réel.

Le deuxième type de problème est d'ordre pratique. On peut le résumer simplement par la question: "Comment utiliser au mieux le modèle? " Il faut savoir, en fait, que le responsable de la gestion d'un barrage peut intervenir directement sur la commande par le biais de la boucle ouverte. C'est ainsi que toute augmentation des "coefficients d'irrigation" (en clair des prélèvements prévus) entraîne une augmentation correspondante de la boucle ouverte, donc de la commande.

Ces interventions sont indispensables en début de campagne quand lâchures et prélèvements sont nuls et qu'on prévoit un démarrage de l'irrigation dans les jours à venir. Ce démarrage est en effet brutal et s'il n'est pas anticipé par le gestionnaire, on peut être sûr de l'assèchement de la rivière. De même, après une pluie, lorsque les irrigations sont arrêtées pour quelques jours, il est plus efficace de donner directement l'information par la modification des coefficients d'irrigation de la boucle ouverte plutôt que d'attendre une réaction de la boucle fermée, toujours plus lente.

Entre ces cas extrêmes, particulièrement évidents, il existe en revanche toute la gamme des cas intermédiaires où la réaction de gestionnaire se superpose avec la réaction en cours de la boucle fermée.
Disons en résumé que, compte tenu de leurs caractéristiques (absence de débits naturels, débit de salubrité faible devant les prélèvements), les rivières réalimentées par la CACG ne peuvent se "piloter" que de façon semiautomatique : ce pilotage est délicat. Par contre, dans le cas de rivières, qui ressemblent plus à des rivières et moins à des canaux (avec un peu de débit naturel et une consigne de salubrité de l'ordre de grandeur des prélèvements), la gestion peut se faire de façon entièrement automatique à condition d'accepter de voir les débits mesurés à l'aval osciller autour de la valeur consigne.

\section{Conclusion}

La distribution de l'eau par les rivières, lorsqu'elle est possible, est sans conteste, financièrement la plus économique. Depuis quelques années, grâce au développement des systèmes d'information et de commande en temps réel, grâce aussi à la mise au point de modèles sans cesse plus performants, elle s'avère aussi être intéressante sur le plan de l'économie... d'eau.

\author{
Auteurs : \\ Patrick Hurand \\ Ingénieur du Génie Rural \\ des Eaux et des Forêts, \\ Compagnie d'Aménagement \\ des Côteaux de Gascogne, \\ Chemin de l'Alette, \\ B.P. 449,65004 Tarbes Cedex. \\ Tél. : 62517220 .
}

Pascal Kosuth, Ingénieur du Génie Rural, des Eaux et des Forêts, CEMAGREF, Domaine Lavalette, av. Val Montferrand, 34000 Montpellier. Tél. : 67524343 . 


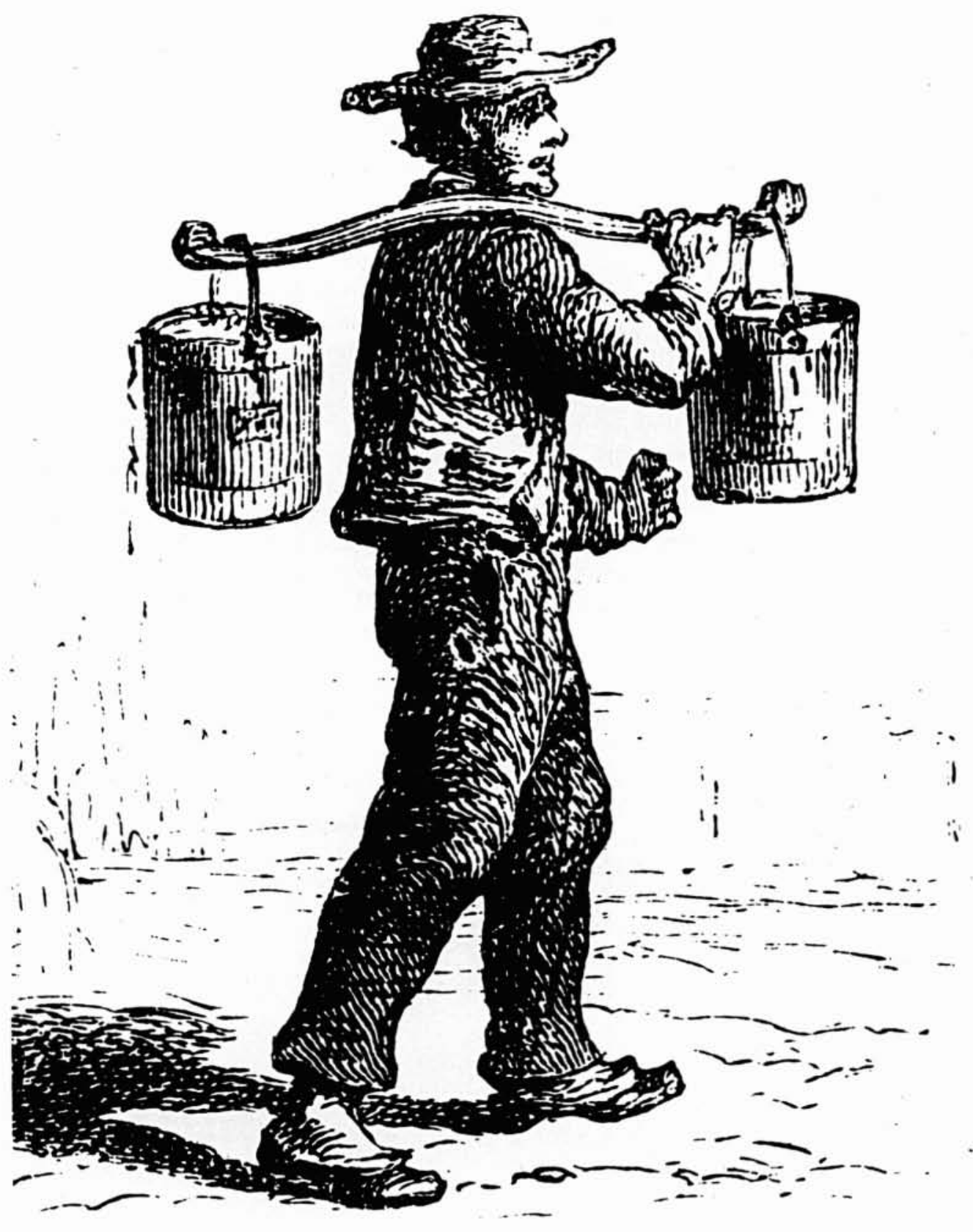

